\title{
Utility of Styrylpyrazoloformimidate in the Synthesis of Fused Heterocyclic Compounds
}

\author{
Hamdi M. Hassaneen*, Zakaria Ahmed Gomaa \\ Department of Chemistry, Faculty of Science, Cairo University, Giza, Egypt \\ Email: "hamdi_251@yahoo.com
}

Received 5 February 2014; accepted 21 November 2015; published 24 November 2015

Copyright $\odot 2015$ by authors and Scientific Research Publishing Inc.

This work is licensed under the Creative Commons Attribution International License (CC BY). http://creativecommons.org/licenses/by/4.0/

(c) (i) Open Access

\begin{abstract}
Refluxing of $(E)$-5-amino-1-phenyl-3-styryl-1H-pyrazole-4-carbonitrile 2 with triethylorthoformate in acetic anhydride afforded the corresponding formimidate 3 . Treatment of 3 with hydrazine hydrate in ethanol afforded amino imino compound 4. Reaction of 4 with diethyl dicarbonate at reflux gave $(E)$-7-phenyl-9-styryl-7H-pyrazolo[4,3-e][1,2,4]triazolo[1,5-c]pyrimidine 7. Refluxing of 4 with hydrazine hydrate afforded $(E)$-4-hydrazinyl-1-phenyl-3-styryl-1H-pyrazolo[3,4-d] pyrimidine 8. Treatment of the latter compound 8 with aldehydes in boiling ethanol in the presence of acetic acid afforded the corresponding hydrazone 10. Oxidative cyclization of the hydrazone 10 led to the formation of pyrazolo $[4,3-e][1,2,4]$ triazolo[ $[4,3-c]$ pyrimidine 11 . The latter products rearranged to pyrazolo[4,3-e][1,2,4]triazolo[1,5-c]pyrimidines 13 . The structures of the new products were established on the basis of elemental analysis and spectral data.
\end{abstract}

\section{Keywords}

(Z)-N'-Phenylcinnamohydrazonoyl Chloride,

(E)-5-Amino-1-Phenyl-3-Styryl-1H-Pyrazole-4-Carbonitrile, Formimidate,

Dimroth Rearrangement

\section{Introduction}

The chemistry of hydrazonoyl halides has attracted the interest of many research groups as they have proved to be useful organic synthesis [1]-[10]. In continuation of our long standing interest for the utility of nitrilimines derived from hydrazonoyl halides in the synthesis of heterocycles [11]-[14], we are interested in (Z)-N'-phenylcinnamohydrazonoyl chloride $\mathbf{1}$ to study the effect of $\mathrm{C}=\mathrm{C}$ double bond on the cycloaddition reactions [15]-[17]. We wish to report herein a simple and convenient route for the synthesis of pyrazolo[3,4- $d$ ]pyrimidine, pyrazo-

*Corresponding author.

How to cite this paper: Hassaneen, H.M. and Gomaa, Z.A. (2015) Utility of Styrylpyrazoloformimidate in the Synthesis of Fused Heterocyclic Compounds. International Journal of Organic Chemistry, 5, 213-222.

http://dx.doi.org/10.4236/ijoc.2015.54021 
lo[4,3-e][1,2,4]triazolo[4,3-c]pyrimidines and its isomeric pyrazolo[4,3-e][1,2,4]triazolo[1,5-c]pyrimidine derivative via Dimroth rearrangement. Such compounds have been used as a new pharmacological test for characterization of human $\mathrm{A}_{3}$ adenosine receptors [18]-[20].

\section{Results and Discussion}

Compound (E)-5-amino-1-phenyl-3-styryl-1H-pyrazole-4-carbonitrile 2 was prepared from our laboratory via reaction of (Z)-N'-phenylcinnamohydrazonoyl chloride $\mathbf{1}$ with malononitrile in ethanolic sodium ethoxide solution (Scheme 1) [21]. Refluxing of compound 2 with triethylorthoformate in acetic anhydride afforded ethyl N-(4-cyano-1-phenyl-3-((E)-styryl)-1H-pyrazol-5-yl)formimidate 3 (Scheme 1). The structure of compound 3 was established on the basis of elemental analysis and spectral data. The IR spectrum of $\mathbf{3}$ revealed the absence of amino group, while it showed a characteristic band at $v 2215 \mathrm{~cm}^{-1}$ assignable to cyano group. Its ${ }^{1} \mathrm{H}$ NMR data showed signals at $\delta, 1.29\left(\mathrm{t}, 3 \mathrm{H}, \mathrm{CH}_{3}\right), 4.32\left(\mathrm{q}, 2 \mathrm{H}, \mathrm{CH}_{2}\right), 7.15-7.69(\mathrm{~m}, 12 \mathrm{H}, \mathrm{Ar} \mathrm{H})$, and $8.60(\mathrm{~s}, 1 \mathrm{H}$, $\mathrm{NCH}$ ). Also, its ${ }^{13} \mathrm{C}-\mathrm{NMR}$ spectrum showed 17 carbon atoms. Moreover, the mass spectrum showed molecular ion peak as a base peak at $\mathrm{m} / \mathrm{z} 342(100 \%)$. Reaction of 3 with hydrazine hydrate in ethanol at room temperature yielded a product 4 which analyzed correctly for $\mathrm{C}_{19} \mathrm{H}_{16} \mathrm{~N}_{6}$ (Scheme 1). The IR spectrum of 4 showed the absence of cyano group and it showed bands at $v 3351,3309,3177 \mathrm{~cm}^{-1}$ assignable to amino and imino groups. Also, the mass spectrum revealed a base peak at $\mathrm{m} / \mathrm{z} 328(100 \%)$ corresponding to its molecular ion peak. On the basis of elemental analysis and spectral data, the product is (E)-4-imino-1-phenyl-3-styryl-1H-pyrazolo [3,4-d]pyrimidin-5(4H)-amine 4.

When compound 4 was refluxed with diethyl dicarbonate a single product was obtained, its mass spectrum and elemental analysis are consistent with the molecular formula $\mathrm{C}_{23} \mathrm{H}_{18} \mathrm{~N}_{6} \mathrm{O}_{2}$ (Scheme 2). Two possible structures were proposed for the isolated product $\mathbf{5 a}$ and $\mathbf{5 b}$. The identity of the isolated product was confirmed to be (E)-ethyl-7-phenyl-9-styryl-7H-pyrazolo[4,3-e][1,2,4]triazolo[1,5-c]pyrimidine-2-carboxylate 5b. Thus, saponification of the reaction product obtained $5 \mathbf{b}$ gave the intermediate acid $\mathbf{6}$ which decarboxylated to a product identical to all respects (m.p., mixed m.p., IR) with (E)-7-phenyl-9-styryl-7H-pyrazolo[4,3-e][1,2,4]triazolo[1,5-c]pyrimidine 7 . The latter product 7 was also confirmed via its alternative synthesis by treatment of compound $\mathbf{4}$ with triethylorthoformate or formic acid. Structure of $\mathbf{5 a}$ was accordingly discarded. In addition, structure 5b was further substantiated by IR and ${ }^{1} \mathrm{H}$ NMR spectra. Its IR spectrum exhibits a carbonyl band at $v 1743$ $\mathrm{cm}^{-1}$ and ${ }^{1} \mathrm{H}$ NMR spectrum showed signals at: $\delta 1.42(\mathrm{t}, \mathrm{J}=7 \mathrm{~Hz}, 3 \mathrm{H}), 4.49(\mathrm{q}, \mathrm{J}=7 \mathrm{~Hz}, 2 \mathrm{H}), 7.37-8.78(\mathrm{~m}, \mathrm{~J}=$ $7 \mathrm{~Hz}, 12 \mathrm{H}$ ), and 9.84 (s, $1 \mathrm{H}$, pyrimidine- $\mathrm{CH}$ ).

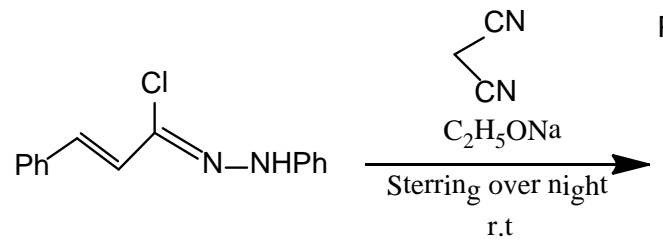

1

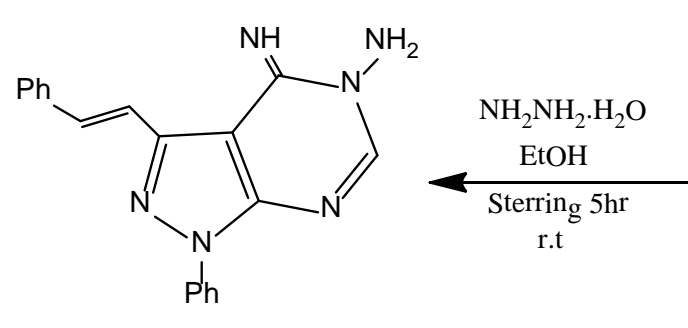

4<smiles>N#Cc1c(/C=C/c2ccccc2)nn(-c2ccccc2)c1N</smiles>

2

Scheme 1. Synthesis of formimidate 3 and amino imino compound 4. 


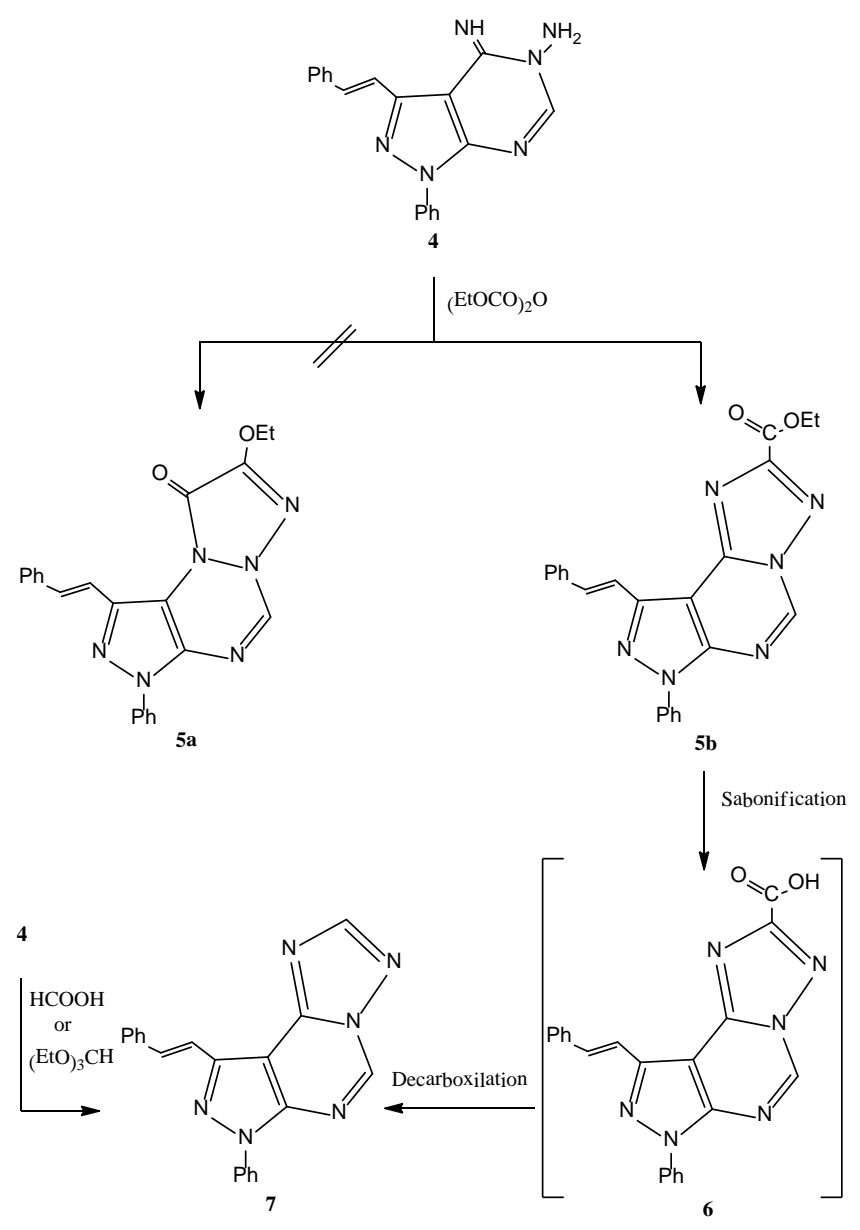

Scheme 2. Synthesis of pyrazolotriazolopyrimidine 7.

Refluxing of compound 4 with hydrazine hydrate in ethanol, gave (E)-4-hydrazinyl-1-phenyl-3-styryl-1Hpyrazolo[3,4-d]pyrimidine 8, via Dimroth type rearrangement, which has not been reported hitherto (Scheme 3 and Scheme 4). This is consistent with a similar rearrangement that was reported recently [22]. The structure of 8 was confirmed by elemental and spectral data (see experimental) and its reaction described below.

Thus, treatment of hydrazine derivative $\mathbf{8}$ with the appropriate aldehydes 9a-i in refluxing ethanol in the presence of acetic acid led to the formation of the new condensation products, 4-(2-arylhydrazinyl)-1-phenyl -3-styryl-1H-pyrazolo[3,4- $d$ ]pyrimidine 10a-i. The structures of 10a-i were confirmed by their elemental analysis and spectral data. For example their IR spectra showed the characteristic band for NH at v $3199-3352 \mathrm{~cm}^{-1}$. Also, their ${ }^{1} \mathrm{H}$ NMR spectra revealed in each case, a signal in the region 11.97 - 12.13 assignable to $\mathrm{NH}$ proton which disappeared upon shaking its DMSO solution with $\mathrm{D}_{2} \mathrm{O}$.

Oxidative cyclization of hydrazone 10a-h led to the formation of pyrazolo[4,3-e][1,2,4]triazolo[4,3-c]pyrimidine derivatives 11a-h (Scheme 3 and Scheme 5). Thus, stirring of 10a-h with 4 equivalent of $\mathrm{Fe}(\mathrm{III})$ chloride in ethanol overnight gave, in each case, a single product as evidenced by TLC analysis. Mass spectra revealed that, each product has 2 hydrogen atoms less than that of the respective hydrazone. Also, IR and ${ }^{1} \mathrm{H}$ NMR revealed the absence of $\mathrm{NH}$ band and $\mathrm{CH}=\mathrm{N}$ proton, respectively.

Compounds 11a,e were isomerized to the thermodynamically more stable pyrazolo[4,3-e][1,2,4]triazolo[1,5-c]pyrimidine derivatives 13a,e through tandem ring opening and ring closure reactions via heating of 11a,e in ethanol in the presence of sodium acetate (Scheme 3). This rearrangement is consistent with those reported in earlier reports [23]. The structures of 13a,e were established by elemental and spectral analysis (see experimental). Also, the structures of 13a,e were confirmed via their alternative synthesis. Thus, treatment of $\mathbf{4}$ with acid chlorides 12a,e in refluxing pyridine gave a products identical in all respects (m.p., mixed m.p., IR and ${ }^{1} \mathrm{H}$ NMR spectra) with those obtained above from base-catalyzed rearrangement of 11a,e (Scheme 3). Also, 


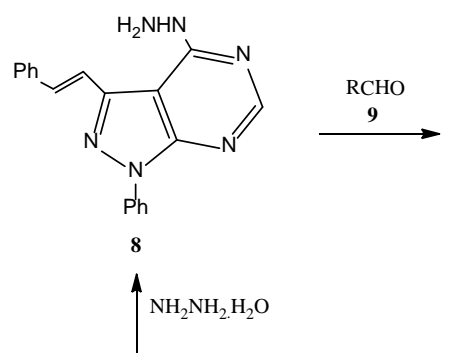<smiles>O=CNc1ncnc2c1c(/C=C/c1ccccc1)nn2-c1ccccc1</smiles>

10

$\mathrm{FeCl}_{3}$<smiles>[R]c1nnc2c3c(/C=C/c4ccccc4)nn(-c4ccccc4)c3ncn12</smiles>

11<smiles>N=c1c2c(/C=C/c3ccccc3)nn(-c3ccccc3)c2ncn1N</smiles>

4

RCOCl<smiles>[R]c1nc2c3c(/C=C/c4ccccc4)nn(-c4ccccc4)c3ncn2n1</smiles>

13

R: a) $\left.\left.\left.=\mathrm{Ph} ; \mathrm{b})=3-\mathrm{CH}_{3} \mathrm{C}_{6} \mathrm{H}_{4} ; \mathrm{c}\right)=4-\left(\mathrm{CH}_{3}\right)_{2} \mathrm{NC}_{6} \mathrm{H}_{4} ; \mathrm{d}\right)=4-\mathrm{OHC}_{6} \mathrm{H}_{4} ; \mathrm{e}\right)=4-\mathrm{ClC}_{6} \mathrm{H}_{4} ;$

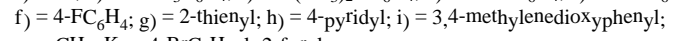
j) $\left.=\mathrm{CH}_{3} ; \mathrm{K}\right)=4-\mathrm{BrC}_{6} \mathrm{H}_{4} ; \mathrm{l}$ ) 2-furyl.

Scheme 3. Synthesis of hydrazinylpyrazolo pyrimidine 8, arylhydrazinylpyrazolopyrimidine 10 and pyrazolotriazolopyrimidine derivatives 11,13 .
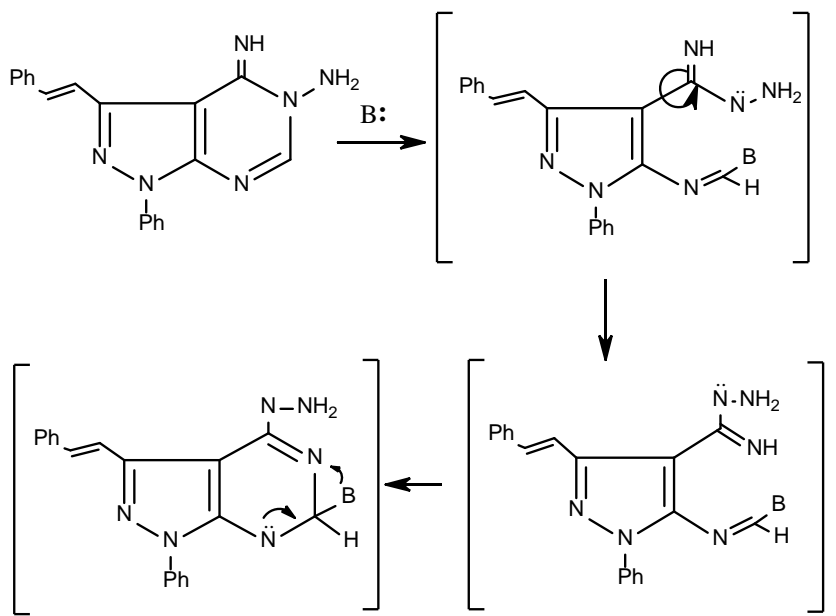

$-: \mathrm{B} \downarrow$<smiles>NNc1ncnc2c1c(/C=C/c1ccccc1)nn2-c1ccccc1</smiles>

Scheme 4. Synthesis of (E)-4-hydrazinyl-1-phenyl-3-styryl-1H-pyrazolo $[3,4-d]$ pyrimidine. 

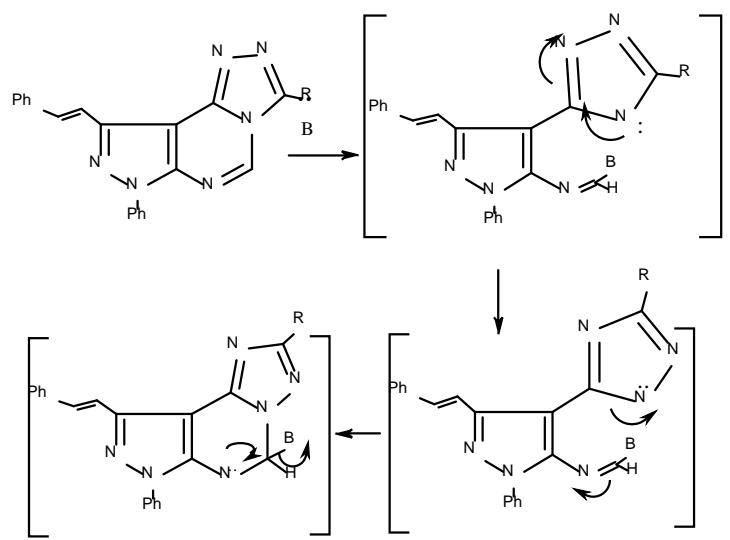<smiles></smiles>

Scheme 5. Synthesis of (E)-2-alkyl-7-phenyl-9-styryl-7H-pyrazolo[4,3-e][1,2,4] triazolo[1,5-c]pyrimidine.

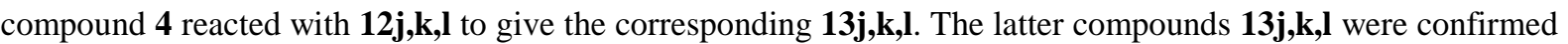
by elemental and spectral analysis.

\section{Experimental}

\subsection{General}

All melting points were determined on an electrothermal GallenKamp melting point apparatus and are uncorrected. The IR spectra were recorded as KBr Pellets on a Jasco FTIR-460 plus Fourier transform infrared spectrophotometer. ${ }^{1} \mathrm{H}$ and ${ }^{13} \mathrm{C}$ NMR spectra were recorded at $(300 \mathrm{MHz})$ and $(75 \mathrm{MHz})$ respectively on Varian EM-300 MHz spectrometer. Chemical shifts $(\delta)$ are given from TMS (ppm) as internal standard for ${ }^{1} \mathrm{H}$ NMR and ${ }^{13} \mathrm{C}$ NMR. Mass spectra were recorded on AEI MS 30 mass spectrometer operating at $70 \mathrm{eV}$. The elemental analyses were performed at the Microanalytical Center of Cairo University. Compound $\mathbf{2}$ was prepared as previously described [18].

\subsection{Preparation of}

Ethyl N-(4-Cyano-1-Phenyl-3-((E)-Styryl)-1H-Pyrazol-5-yl)Formimidate 3

To a solution of the compound (E)-5-amino-1-phenyl-3-styryl-1H-pyrazole-4-carbonitrile 2 (1.43 g, 5 mmol) in acetic anhydride $(5 \mathrm{~mL})$, triethylorthoformate $(0.74 \mathrm{~g}, 5 \mathrm{mmol})$ was added. The reaction mixture was refluxed for $5 \mathrm{~h}$ and the solvent was evaporated under reduced pressure. The solid product was collected and crystallized from acetonitrile to afford the compound 3. Yellow crystals; m.p: $170^{\circ} \mathrm{C}-171^{\circ} \mathrm{C}$; yield $(82 \%)$; IR $(\mathrm{KBr}): v=$ 2215 (CN) cm ${ }^{-1}$; ${ }^{1} \mathrm{H}$ NMR (DMSO-d6): $\delta=1.29$ (t, 3H, $\mathrm{CH}_{2} \mathrm{CH}_{3}$ ), 4.32 (q, 2H, $\mathrm{CH}_{3} \mathrm{CH}_{2}$ ), 7.15 - 7.69 (m, 12H, Ar H), 8.60 (s, $1 \mathrm{H}, \mathrm{NCH}$ ); ${ }^{13} \mathrm{C}$ NMR (DMSO-d6): $\delta=13.74,64.07,79.46,114.46,117.78,123.78,126.88$, 128.00, 128.74, 128.85, 128.97, 132.88, 135.58, 137.46, 149.57, 151.51, 162.46. MS: $\mathrm{m} / \mathrm{z}(\%)=342\left(\mathrm{M}^{+}, 100\right)$, 313 (31), 285 (45), 77 (41). Anal. for $\mathrm{C}_{21} \mathrm{H}_{18} \mathrm{~N}_{4} \mathrm{O}$ : Calcd. C, 73.67; H, 5.30; N, 16.36. found C, 73.31; H, 5.03; N, 16.11 .

\subsection{Preparation of}

(E)-4-Imino-1-Phenyl-3-Styryl-1H-Pyrazolo[3,4- $d]$ Pyrimidin-5(4H)-Amine 4

To a solution of the compound 3 (17.1 g, $50 \mathrm{mmol})$ in ethanol $(250 \mathrm{~mL})$, hydrazine hydrate $(2.5 \mathrm{~mL}, 50 \mathrm{mmol})$ 
was added. The reaction mixture was stirred for $5 \mathrm{~h}$ at room temperature; the solid product was collected and crystallized from dioxane to afford the compound 4. Yellow crystals; m.p. $200^{\circ} \mathrm{C}-202^{\circ} \mathrm{C}$; yield (91\%); IR $(\mathrm{KBr}): v=3351 \& 3309\left(\mathrm{NH}_{2}\right), 3177(\mathrm{NH}) \mathrm{cm}^{-1}$. MS: $\mathrm{m} / \mathrm{z}(\%)=328\left(\mathrm{M}^{+}, 100\right), 313$ (29), 77 (30). Anal. for $\mathrm{C}_{19} \mathrm{H}_{16} \mathrm{~N}_{6}$ : Calcd. C, 69.50; H, 4.91; N, 25.59. found C, 69.07; H, 4.72; N, 25.39 .

\subsection{Preparation of \\ (E)-9-Styryl-7H-Pyrazolo[4,3-e][1,2,4]Triazolo[1,5-c]Pyrimidine-2-Carboxylate Ethyl 7-Phenyl 5b}

A solution of the compound 4 (1.64 g, $5 \mathrm{mmol})$ in diethyl dicarbonate $(10 \mathrm{~mL})$ was refluxed for $\mathbf{4} \mathrm{h}$. and then cooled. The solid product was filtered off, dried and finally crystallized from acetic acid to give $\mathbf{5 b}$. Yellow crystals; m.p. $212^{\circ} \mathrm{C}-214^{\circ} \mathrm{C}$; yield (88\%); IR (KBr): $v=1743$ (CO) $\mathrm{cm}^{-1} ;{ }^{1} \mathrm{H}$ NMR (DMSO-d6): $\delta=1.42$ (t, 3H, $\mathrm{CH}_{2} \mathrm{CH}_{3}$ ), 4.49 (q, $2 \mathrm{H}, \mathrm{CH}_{3} \mathrm{CH}_{2}$ ), 7.37 - 8.78 (m, $\left.12 \mathrm{H}, \mathrm{Ar} \mathrm{H}\right), 9.84$ (s, $\left.1 \mathrm{H}, \mathrm{NCH}\right) . \mathrm{MS}: \mathrm{m} / \mathrm{z}(\%)=410(\mathrm{M}+, 96)$, 409 (50), 77 (100). Anal. for $\mathrm{C}_{23} \mathrm{H}_{18} \mathrm{~N}_{6} \mathrm{O}_{2}$ : Calcd. C, 67.31; H, 4.42; N, 20.48. found C, 67.02; H, 4.37; N, 20.25.

\subsection{Preparation of \\ (E)-7-Phenyl-9-Styryl-7H-Pyrazolo[4,3-e][1,2,4]Triazolo[1,5-c]Pyrimidine 7}

A solution of the compound 4 (1.64 g, $5 \mathrm{mmol})$ in triethylorthoformate or formic acid (10 mL) was refluxed for $4 \mathrm{~h}$. left to cool and the solid product was filtered, dried and finally crystallized from acetic acid to afford 7 . Pale yellow crystals; m.p. $198^{\circ} \mathrm{C}-200^{\circ} \mathrm{C}$ (acetic acid); yield (86\%); ${ }^{1} \mathrm{H}$ NMR (DMSO- $d 6$ ): $\delta=6.98-8.56$ (m, $12 \mathrm{H}$, Ar H), 9.77 (s, 1H, NCH), 9.79 (s, 1H, NCH). MS: $m / z(\%)=338\left(\mathrm{M}^{+}, 98\right), 337$ (100), 77 (30). Anal. for $\mathrm{C}_{20} \mathrm{H}_{14} \mathrm{~N}_{6}$ : Calcd. C, 70.99; H, 4.17; N, 24.84. found C, 70.65; H, 4.12; N, 24.61.

\subsection{Preparation of (E)-4-Hydrazinyl-1-Phenyl-3-Styryl-1H-Pyrazolo[3,4- $d]$ Pyrimidine 8}

A solution of the compound $4(16.4 \mathrm{~g}, 50 \mathrm{mmol})$ in ethanol $(250 \mathrm{~mL})$ and hydrazine hydrate $(10 \mathrm{~mL})$ was refluxed for $5 \mathrm{~h}$. The solvent was evaporated; the solid product was collected, dried and finally crystallized from acetonitrile to afford 8. Yellow crystals; m.p. $202^{\circ} \mathrm{C}-204^{\circ} \mathrm{C}$; yield $(90 \%)$; IR $(\mathrm{KBr}): v=3310(\mathrm{NH}), 3351$ \& $3309\left(\mathrm{NH}_{2}\right) \mathrm{cm}^{-1}$; ${ }^{1} \mathrm{H}$ NMR (DMSO-d6): $\delta=4.86$ (s, $2 \mathrm{H}, \mathrm{NH}_{2}$ ), 7.31-8.41 (m, 13H, Ar H), 9.25 (s, $\left.1 \mathrm{H}, \mathrm{NH}\right) . \mathrm{MS}$ : $\mathrm{m} / \mathrm{z}(\%)=\left(\mathrm{M}^{+}, 38\right), 313$ (30), 297 (80), 77 (100). Anal. for $\mathrm{C}_{19} \mathrm{H}_{16} \mathrm{~N}_{6}$ : Calcd. C, 69.50; H, 4.91; N, 25.59. found C, 69.22; H, 4.81; N, 25.33.

\subsection{General Method for Preparation}

\section{(E)-4-(2-Arylhydrazinyl)-1-Phenyl-3-Styryl-1H-Pyrazolo[3,4- $d]$ Pyrimidine 10a-i}

To a mixture of compound 8 (1.64 g, $5 \mathrm{mmol})$ in ethanol (30 mL), the appropriate aldehyde 9a-i (5 mmol) was added. The reaction mixture was refluxed for $2 \mathrm{~h}$ and the solvent was evaporated. The solid product formed was collected, dried and finally crystallized from suitable solvent to afford 10a-i.

4-((Z)-2-Benzylidenehydrazinyl)-1-phenyl-3-((E)-styryl)-1H-pyrazolo[3,4-d]pyrimidine 10a:

Yellow crystals; m.p. $188^{\circ} \mathrm{C}-189^{\circ} \mathrm{C}$ (acetonitrile); yield (87\%); IR (KBr): v $=3205(\mathrm{NH}) \mathrm{cm}^{-1},{ }^{1} \mathrm{H}$ NMR (DMSO-d6): $\delta=7.15$ - 8.58 (m, 19H, Ar H), 12.04 (s, 1H, NH). MS: m/z (\%) = $416\left(\mathrm{M}^{+}, 14\right), 415$ (42), 313 (43), 236 (41), 77 (100). Anal. for $\mathrm{C}_{26} \mathrm{H}_{20} \mathrm{~N}_{6}$ : Calcd. C, 74.98; H, 4.84; N, 20.18. found C, 74.37; H, 4.71; N, 19.73.

4-((Z)-2-(3-Methylbenzylidene)hydrazinyl)-1-phenyl-3-((E)-styryl)-1H-pyrazolo[3,4-d]pyrimidine 10b:

Yellow crystals; m.p. $192^{\circ} \mathrm{C}-194^{\circ} \mathrm{C}$ (acetonitrile); yield $(81 \%)$; IR $(\mathrm{KBr}): v=3352(\mathrm{NH}) \mathrm{cm}^{-1},{ }^{1} \mathrm{H}$ NMR (DMSO-d6): $\delta=2.36$ (s, 3H, $\mathrm{CH}_{3}$ ), 7.24-8.52 (m, 18H, Ar H), 12.01 (s, 1H, NH); ${ }^{13} \mathrm{C}$ NMR (DMSO-d6): $\delta=$ 20.83, 99.47, 119.11, 121.83, 125.20, 126.783, 128.18, 128.41, 128.76, 129.01, 130.57, 132.80, 136.52, 137.80, 138.19, 147.89, 153.98. MS: $m / z(\%)=430$ ( $\left.\mathrm{M}^{+}, 79\right), 339$ (52), 312 (100), 236 (79), 77 (80). Anal. for $\mathrm{C}_{27} \mathrm{H}_{22} \mathrm{~N}_{6}$ : Calcd. C, 75.33; H, 5.15; N, 19.52. found C, 75.06; H, 4.92; N, 19.36.

N,N-Dimethyl-4-((Z)-(2-(1-phenyl-3-((E)-styryl)-1H-pyrazolo[3,4-d]pyrimidin-4-yl)hydrazono)methyl)aniline 10c:

Yellow crystals; m.p. $242^{\circ} \mathrm{C}-244^{\circ} \mathrm{C}$ (dimethylformamide); yield (77\%); IR (KBr): v $=3199(\mathrm{NH}) \mathrm{cm}^{-1} ;{ }^{1} \mathrm{H}$ NMR (DMSO-d6): $\delta=2.99$ (s, 6H, N( $\left.\left(\mathrm{CH}_{3}\right)_{2}\right), 6.75-8.43$ (m, 18H, Ar H), 11.87 (s, 1H, NH). MS: $\mathrm{m} / \mathrm{z}(\%)=459$ ( $\left.\mathrm{M}^{+}, 34\right), 457$ (62), 312 (100), 236 (65), 145 (60), 77 (91). Anal. for $\mathrm{C}_{28} \mathrm{H}_{25} \mathrm{~N}_{7}$ : Calcd. C, 73.18; H, 5.48; N, 21.34. found C, 72.55; H, 5.23; N, 20.84. 
4-((Z)-(2-(1-Phenyl-3-((E)-styryl)-1H-pyrazolo[3,4-d]pyrimidin-4-yl)hydrazono)methyl)phenol 10d:

Yellow crystals; m.p. $270^{\circ} \mathrm{C}-272^{\circ} \mathrm{C}$ (acetonitrile); yield (83\%); IR $(\mathrm{KBr}): v=3324(\mathrm{NH}) \mathrm{cm}^{-1} ;{ }^{1} \mathrm{H}$ NMR (DMSO-d6): $\delta=6.99$ - 8.68 (m, 18H, Ar H), 11.98 (s, 1H, NH), 13.02 (s, 1H, OH). MS: $\mathrm{m} / \mathrm{z}(\%)=432\left(\mathrm{M}^{+}, 82\right)$, 312 (100), 77 (93). Anal. for $\mathrm{C}_{26} \mathrm{H}_{20} \mathrm{~N}_{6} \mathrm{O}$ : Calcd. C, 72.21; H, 4.66; N, 19.43. found C, 71.76; H, 4.51; N, 19.11.

4-((Z)-2-(4-Chlorobenzylidene)hydrazinyl)-1-phenyl-3-((E)-styryl)-1H-pyrazolo[3,4-d]pyrimidine 10e:

Yellow crystals; m.p. $230^{\circ} \mathrm{C}-232^{\circ} \mathrm{C}$ (dimethylformamide); yield (84\%); IR (KBr): $v=3205(\mathrm{NH}) \mathrm{cm}^{-1} ;{ }^{1} \mathrm{H}$ NMR (DMSO-d6): $\delta=7.02-8.70$ (m, 18H, Ar H), 12.11 (s, 1H, NH). MS: $m / z(\%)=450\left(\mathrm{M}^{+}, 100\right), 77$ (72). Anal. for $\mathrm{C}_{26} \mathrm{H}_{19} \mathrm{ClN}_{6}$ : Calcd. C, 69.25; H, 4.25; Cl, 7.86; N, 18.64. found C, 68.76; H, 4.03; Cl, 7.65; N, 18.23.

4-((Z)-2-(4-Fluorobenzylidene)hydrazinyl)-1-phenyl-3-((E)-styryl)-1H-pyrazolo[3,4-d]pyrimidine 10f:

Yellow crystals; m.p. $192^{\circ} \mathrm{C}-194^{\circ} \mathrm{C}$ (acetonitrile); yield (87\%); IR (KBr): v $=3199(\mathrm{NH}) \mathrm{cm}^{-1} ;{ }^{1} \mathrm{H} \mathrm{NMR}$ (DMSO-d6): $\delta=7.04-8.71$ (m, 18H, Ar H), 12.13 (s, 1H, NH). MS: m/z (\%) = $434\left(\mathrm{M}^{+}, 87\right), 339$ (32), 312 (100), 236 (79), 77 (56). Anal. for $\mathrm{C}_{26} \mathrm{H}_{19} \mathrm{FN}_{6}$ : Calcd. C, 71.88; H, 4.41; F, 4.37; N, 19.34. found C, 71.51; H, 4.30; F, 4.29; N, 19.03.

1-Phenyl-3-((E)-styryl)-4-((Z)-2-(thiophen-2-ylmethylene)hydrazinyl)-1H-pyrazolo[3,4-d]pyrimidine 10g:

Yellow crystals; m.p. $186^{\circ} \mathrm{C}-188^{\circ} \mathrm{C}$ (acetonitrile); yield $(83 \%)$; IR $(\mathrm{KBr}): v=3337(\mathrm{NH}) \mathrm{cm}^{-1} ;{ }^{1} \mathrm{H}$ NMR (DMSO-d6): $\delta=7.01-8.71$ (m, 17H, Ar H), 12.01 (s, 1H, NH). MS: m/z (\%) = $422\left(\mathrm{M}^{+}, 100\right), 312$ (88), 236 (61), 77 (42). Anal. for $\mathrm{C}_{24} \mathrm{H}_{18} \mathrm{~N}_{6} \mathrm{~S}$ : Calcd. C, 68.23; H, 4.29; N, 19.89; S, 7.59. found C, 67.81; H, 4.20; N, 19.64; S, 7.50.

1-Phenyl-4-((Z)-2-(pyridin-4-ylmethylene)hydrazinyl)-3-((E)-styryl)-1H-pyrazolo[3,4-d]pyrimidine 10h:

Yellow crystals; m.p. $284^{\circ} \mathrm{C}-286^{\circ} \mathrm{C}$ (dimethylformamide); yield $(84 \%) ; \mathrm{IR}(\mathrm{KBr}): \mathrm{v}=3322(\mathrm{NH}) \mathrm{cm}^{-1} ;{ }^{1} \mathrm{H}$ NMR (DMSO-d6): $\delta=6.89-8.64$ (m, 18H, Ar H), 11.97 (s, 1H, NH). MS: $m / z(\%)=417\left(\mathrm{M}^{+}, 54\right), 312(71)$, 77 (100). Anal. for $\mathrm{C}_{25} \mathrm{H}_{19} \mathrm{~N}_{7}$ : Calcd. C, 71.93; H, 4.59; N, 23.49. found C, 71.58; H, 4.24; N, 23.11.

4-((Z)-2-(Benzo[d][1,3]dioxol-5-ylmethylene)hydrazinyl)-1-phenyl-3-((E)-styryl)-1H-pyrazolo[3,4-d]pyrimidine 10i:

Yellow crystals; m.p. $214^{\circ} \mathrm{C}-216^{\circ} \mathrm{C}$ (acetonitrile); yield $(82 \%)$; IR $(\mathrm{KBr}): \mathrm{v}=3341(\mathrm{NH}) \mathrm{cm}^{-1} ;{ }^{1} \mathrm{H}$ NMR (DMSO-d6): $\delta=6.09$ (s, 2H, CH CH $_{2} 6.97-8.47$ (m, 17H, Ar H), 12.01 (s, $\left.1 \mathrm{H}, \mathrm{NH}\right) . \mathrm{MS}: \mathrm{m} / \mathrm{z}(\%)=460\left(\mathrm{M}^{+}, 54\right)$, 312 (100), 236 (63), 77 (92). Anal. for $\mathrm{C}_{27} \mathrm{H}_{20} \mathrm{~N}_{6} \mathrm{O}_{2}$ : Calcd. C, 70.42; H, 4.38; N, 18.25. found C, 70.01; H, 4.30; N, 18.11.

\subsection{General Method for Preparation of 7H-Pyrazolo[4,3-e][1,2,4]Triazolo[4,3-c] Pyrimidine 11a-h}

To a solution of an appropriate arylhydrazinyl compound 10a-h (5 mmol) in ethanol (20 mL), ferric chloride (4 $\mathrm{mL}, 2 \mathrm{M}$ ) was added, and the reaction mixture was stirred for $24 \mathrm{~h}$. The solid that separated was collected, dried and finally crystallized from dimethylformamide to afford the corresponding 7H-pyrazolo[4,3-e][1,2,4]triazolo[4,3-c]pyrimidine 11a-h.

(E)-3,7-Diphenyl-9-styryl-7H-pyrazolo[4,3-e][1,2,4]triazolo[4,3-c]pyrimidine 11a:

White crystals; m.p. $276^{\circ} \mathrm{C}-278^{\circ} \mathrm{C}$; yield (87\%); ${ }^{1} \mathrm{H}$ NMR (DMSO-d6): $\delta=7.37$ - 8.81 (m, 17H, Ar H), 9.32 (s, $1 \mathrm{H}, \mathrm{NCH})$. MS: $\mathrm{m} / \mathrm{z}(\%)=414\left(\mathrm{M}^{+}, 100\right), 77$ (80). Anal. for $\mathrm{C}_{26} \mathrm{H}_{18} \mathrm{~N}_{6}$ : Calcd. C, 75.35; H, 4.38; N, 20.28 . found C, 75.03; H, 4.32; N, 20.01.

(E)-7-Phenyl-9-styryl-3-(m-tolyl)-7H-pyrazolo[4,3-e][1,2,4]triazolo[4,3-c]pyrimidine 11b:

White crystals; m.p. $182^{\circ} \mathrm{C}-184^{\circ} \mathrm{C}$; yield (86\%); ${ }^{1} \mathrm{H}$ NMR (DMSO-d6): $\delta=2.23$ (s, 3H, $\mathrm{CH}_{3}$ ), 7.31 - 8.47 (m, 16H, Ar H), 9.08 (s, $1 \mathrm{H}, \mathrm{NCH})$. MS: $\mathrm{m} / \mathrm{z}(\%)=428\left(\mathrm{M}^{+}, 100\right)$, 77 (26). Anal. for $\mathrm{C}_{27} \mathrm{H}_{20} \mathrm{~N}_{6}$ : Calcd. C, 75.68; $\mathrm{H}$, 4.70; N, 19.61. found C, 75.22; H, 4.410; N, 19.15 .

(E)-N,N-Dimethyl-4-(7-phenyl-9-styryl-7H-pyrazolo[4,3-e][1,2,4]triazolo[4,3-c]pyrimidin-3-yl)aniline 11c:

Yellow crystals; m.p. $222^{\circ} \mathrm{C}-224^{\circ} \mathrm{C}$; yield (85\%); ${ }^{1} \mathrm{H}$ NMR (DMSO-d6): $\delta=3.02\left(\mathrm{~s}, 6 \mathrm{H}, \mathrm{N}\left(\mathrm{CH}_{3}\right)_{2}\right), 6.89$ $8.41(\mathrm{~m}, 16 \mathrm{H}, \mathrm{Ar} \mathrm{H}), 9.24(\mathrm{~s}, 1 \mathrm{H}, \mathrm{NCH})$. MS: $\mathrm{m} / \mathrm{z}(\%)=457\left(\mathrm{M}^{+}, 47\right), 77(100)$. Anal. for $\mathrm{C}_{28} \mathrm{H}_{23} \mathrm{~N}_{7}$ : Calcd. C, 73.50; H, 5.07; N, 21.43. found C, 73.21; H, 4.98; N, 21.24.

(E)-4-(7-Phenyl-9-styryl-7H-pyrazolo[4,3-e][1,2,4]triazolo[4,3-c]pyrimidin-3-yl)phenol 11d:

Pale green crystals; m.p. $284^{\circ} \mathrm{C}-286^{\circ} \mathrm{C}$; yield (88\%); ${ }^{1} \mathrm{H}$ NMR (DMSO-d6): $\delta=6.93-8.45$ (m, $16 \mathrm{H}, \mathrm{Ar} \mathrm{H}$ ), 9.28 (s, $1 \mathrm{H}, \mathrm{NCH}), 13.11$ (s, $1 \mathrm{H}, \mathrm{OH})$. MS: $m / z(\%)=430\left(\mathrm{M}^{+}, 18\right), 77$ (100). Anal. for $\mathrm{C}_{26} \mathrm{H}_{18} \mathrm{~N}_{6} \mathrm{O}$ : Calcd. C, 72.55; H, 4.21; N, 19.52. found C, 72.31; H, 4.16; N, 19.39 .

(E)-3-(4-Chlorophenyl)-7-phenyl-9-styryl-7H-pyrazolo[4,3-e][1,2,4]triazolo[4,3-c]pyrimidine 11e: 
Yellow crystals; m.p. $250^{\circ} \mathrm{C}-252^{\circ} \mathrm{C}$; yield (80\%); ${ }^{1} \mathrm{H}$ NMR (DMSO-d6): $\delta=7.33-8.12$ (m, 16H, Ar H), 8.66 (s, $1 \mathrm{H}, \mathrm{NCH}) . \mathrm{MS}: \mathrm{m} / \mathrm{z}(\%)=448\left(\mathrm{M}^{+}, 100\right), 77$ (48). Anal. for $\mathrm{C}_{26} \mathrm{H}_{17} \mathrm{ClN}_{6}$ : Calcd. C, 69.56; H, 3.82; Cl, 7.90; N, 18.72. found C, 69.14; H, 3.75; Cl, 7.78; N, 18.59 .

(E)-3-(4-Fluorophenyl)-7-phenyl-9-styryl-7H-pyrazolo[4,3-e][1,2,4]triazolo[4,3-c]pyrimidine 11f:

White crystals; m.p. $228^{\circ} \mathrm{C}-230^{\circ} \mathrm{C}$; yield (81\%); ${ }^{1} \mathrm{H}$ NMR (DMSO-d6): $\delta=7.34-8.14$ (m, 16H, Ar H), 8.81 (s, $1 \mathrm{H}, \mathrm{NCH})$. MS: $\mathrm{m} / \mathrm{z}(\%)=432\left(\mathrm{M}^{+}, 100\right), 77$ (21). Anal. for $\mathrm{C}_{26} \mathrm{H}_{17} \mathrm{FN}_{6}$ : Calcd. C, 72.21; H, 3.96; F, 4.39; N, 19.43. found C, 71.43; H, 3.90; F, 4.32; N, 19.31 .

(E)-7-Phenyl-9-styryl-3-(thiophen-2-yl)-7H-pyrazolo[4,3-e][1,2,4]triazolo[4,3-c]pyrimidine $11 \mathrm{~g}$ :

Green crystals; m.p. $262^{\circ} \mathrm{C}-264{ }^{\circ} \mathrm{C}$; yield (84\%); ${ }^{1} \mathrm{H}$ NMR (DMSO-d6): $\delta=7.33-8.73$ (m, $\left.15 \mathrm{H}, \mathrm{Ar} \mathrm{H}\right), 9.48$ (s, 1H, NCH); ${ }^{13} \mathrm{C}$ NMR (DMSO-d6): $\delta=98.57,118.90,122.25,126.39,126.81,127.59,128.51,128.88$, 129.24, 129.72, 135.62, 136.38, 137.73, 138.69, 142.28, 144.87, 145.56. MS: m/z (\%) = $420\left(\mathrm{M}^{+}, 100\right), 77$ (18). Anal. for $\mathrm{C}_{24} \mathrm{H}_{16} \mathrm{~N}_{6} \mathrm{~S}$ : Calcd. C, 68.55; H, 3.84; N, 19.99; S, 7.63. found C, 68.01; H, 3.80; N, 19.51; S, 7.52.

(E)-7-Phenyl-3-(pyridin-4-yl)-9-styryl-7H-pyrazolo[4,3-e][1,2,4]triazolo[4,3-c]pyrimidine $11 \mathrm{~h}$ :

Orange crystals; m.p. $268^{\circ} \mathrm{C}-270^{\circ} \mathrm{C}$; yield (83\%); ${ }^{1} \mathrm{H}$ NMR (DMSO-d6): $\delta=6.91-8.68$ (m, 16H, Ar H), 9.36 (s, $1 \mathrm{H}, \mathrm{NCH})$. MS: $m / z(\%)=415\left(\mathrm{M}^{+}, 100\right), 77$ (41). Anal. for $\mathrm{C}_{25} \mathrm{H}_{17} \mathrm{~N}_{7}$ : Calcd. C, 72.28; H, 4.12; N, 23.60. found C, 71.74; H, 4.06; N, 23.31.

\subsection{General Methods for Preparation of Pyrazolo[4,3-e][1,2,4]Triazolo[1,5-c]Pyrimidine Derivatives 13}

Method A: To a solution of the appropriate 11a,e $(1 \mathrm{mmol})$ in absolute ethanol $(30 \mathrm{~mL})$, sodium acetate $(0.16 \mathrm{~g}$, $2 \mathrm{mmol}$ ) was added and the mixture was refluxed for $8 \mathrm{~h}$. The precipitated solid after cooling was filtered, washed with water, dried and finally crystallized from suitable solvent to give the respective products 13a,e.

Method B: To a solution of the compound 4 (1.64 g, $5 \mathrm{mmol})$ in pyridine (20 mL), the appropriate acid chloride 12a,e,j,k,l ( $5 \mathrm{mmol}$ ) was added. The reaction mixture was refluxed for $4 \mathrm{~h}$, then cooled and poured over crushed ice containing hydrochloric acid (10\%) with stirring. The solid product was filtered, washed with water, dried and finally crystallized from suitable solvent to afford 13a,e,j,k,l.

(E)-2,7-Diphenyl-9-styryl-7H-pyrazolo[4,3-e][1,2,4]triazolo[1,5-c]pyrimidine 13a:

Pale brown crystals; m.p. $210^{\circ} \mathrm{C}-212^{\circ} \mathrm{C}$ (dimethylformamide); yield (80\%); ${ }^{1} \mathrm{H}$ NMR (DMSO-d6): $\delta=6.66$ 8.75 (m, 17H, Ar H), 9.78 (s, 1H, NCH). MS: m/z (\%) = $414\left(\mathrm{M}^{+}, 100\right), 77$ (28). Anal. for $\mathrm{C}_{26} \mathrm{H}_{18} \mathrm{~N}_{6}$ : Calcd C, 75.35; H, 4.38; N, 20.28. found C, 74.98; H, 4.18; N, 19.89.

(E)-2-(4-Chlorophenyl)-7-phenyl-9-styryl-7H-pyrazolo[4,3-e][1,2,4]triazolo[1,5-c]pyrimidine 13e:

Pale yellow crystals; m.p. $268^{\circ} \mathrm{C}-270^{\circ} \mathrm{C}$ (dimethylformamide); yield (87\%); ${ }^{1} \mathrm{H}$ NMR (DMSO-d6): $\delta=6.68-$ 8.84 (m, 16H, Ar H), 9.81 (s, 1H, NCH). MS: m/z (\%) = $448\left(\mathrm{M}^{+}, 100\right), 447$ (68), 77 (54). Anal. for $\mathrm{C}_{26} \mathrm{H}_{17} \mathrm{ClN}_{6}$ : Calcd C, 69.56; H, 3.82; Cl, 7.90; N, 18.72. found C, 69.31; H, 3.66; Cl, 7.69; N, 18.40.

(E)-2-Methyl-7-phenyl-9-styryl-7H-pyrazolo[4,3-e][1,2,4]triazolo[1,5-c]pyrimidine 13j:

White crystals; m.p. $186^{\circ} \mathrm{C}-187^{\circ} \mathrm{C}$ (acetic acid); yield (92\%); ${ }^{1} \mathrm{H}$ NMR (DMSO-d6): $\delta=2.57\left(\mathrm{~s}, 3 \mathrm{H}, \mathrm{CH}_{3}\right)$, 7.32 - 8.59 (m, 12H, Ar H), 9.50 (s, 1H, NCH); ${ }^{13} \mathrm{C}$ NMR (DMSO-d6): $\delta=14.28,118.81,122.05,126.82$, 127.37, 128.54, 128.88, 129.22, 135.60, 136.37, 137.87, 140.16, 144.17, 164.41. MS: $\mathrm{m} / \mathrm{z}(\%)=352\left(\mathrm{M}^{+}, 98\right)$, 351 (100), 77 (35). Anal. for $\mathrm{C}_{21} \mathrm{H}_{16} \mathrm{~N}_{6}$ : Calcd. C, 71.58; H, 4.58; N, 23.85. found C, 71.01; H, 4.33; N, 23.52.

(E)-2-(4-Bromophenyl)-7-phenyl-9-styryl-7H-pyrazolo[4,3-e][1,2,4]triazolo[1,5-c]pyrimidine 13k:

Pale yellow crystals; m.p. $290^{\circ} \mathrm{C}-292^{\circ} \mathrm{C}$ (dimethylformamide); yield (85\%); ${ }^{1} \mathrm{H}$ NMR (DMSO-d6): $\delta=6.71-$ 8.80 (m, 16H, Ar H), 9.84 (s, 1H, NCH). MS: m/z (\%) = 492 (M $\left.\mathrm{M}^{+}, 100\right), 491$ (75), 206 (30), 102 (29), 77 (81). Anal. for $\mathrm{C}_{26} \mathrm{H}_{17} \mathrm{BrN}_{6}$ : Calcd C, 63.30; H, 3.47; Br, 16.20; N, 17.03. found C, 63.01; H, 3.39; Br, 16.03; N, 16.86.

(E)-2-(Furan-2-yl)-7-phenyl-9-styryl-7H-pyrazolo[4,3-e][1,2,4]triazolo[1,5-c]pyrimidine 13l:

Pale yellow crystals; m.p. $264^{\circ} \mathrm{C}-266^{\circ} \mathrm{C}$ (dimethylformamide); yield (86\%); ${ }^{1} \mathrm{H}$ NMR (DMSO- $d 6$ ): $\delta=6.75$ 8.77 (m, 15H, Ar H), 9.62 (s, 1H, NCH). MS: m/z (\%) = $404\left(\mathrm{M}^{+}, 100\right), 403$ (65), 77 (53). Anal. for $\mathrm{C}_{24} \mathrm{H}_{16} \mathrm{~N}_{6} \mathrm{O}$ : Calcd C, 71.28; H, 3.99; N, 20.78. found C, 70.79; H, 3.63; N, 20.47.

\section{References}

[1] Wolkoff, P. (1975) A New Method of Preparing Hydrazonoyl Halides. Canadian Journal of Chemistry, 53, $1333-1335$. http://dx.doi.org/10.1139/v75-183 
[2] Shawali, A.S. and Abdel Hamid, A.O. (1976) Reaction of Dimethyiphenacylsulfonium Bromide with $N$-Nitrosoacetarylamides and Reactions of the Products with Nucleophiles. Bulletin of the Chemical Society of Japan, 49, 321. http://dx.doi.org/10.1246/bcsj.49.321

[3] Farag, A.M. and Algharib, M.S. (1988) Synthesis and Reactions of C-(2-Thenoyl)-N-Arylformhydrazidoyl Bromides. Organic Preparations and Procedures International: The New Journal for Organic Synthesis, 20, 521-526. http://dx.doi.org/10.1080/00304948809356298

[4] Eweiss, N.F. and Osman, A. (1980) Synthesis of Heterocycles. Part II. New Routes to Acetylthiadiazolines and Alkylazothiazoles. Journal of Heterocyclic Chemistry, 17, 1713-1717. http://dx.doi.org/10.1002/jhet.5570170814

[5] Hassaneen, H.M., Shawali, A.S., Elwan, N.M. and Abounada, N.M. (1992) Reaction of 1-(2-Naphthoyl)methyl-2-dimethylsulfonium Bromide with $\mathrm{N}$-Nitroso- $\mathrm{N}$-arylacetamides and Reactions of the Products with Some Nucleophiles. Sulfur Letters, 13, 273-285.

[6] Shawali, A.S. and Parkanyi, C. (1980) Hydrazidoyl Halides in the Synthesis of Heterocycles. Journal of Heterocyclic Chemistry, 17, 833-854. http://dx.doi.org/10.1002/jhet.5570170501

[7] Shawali, A.S. and Sherif, S.M. (2007) The Chemistry of Hydrazonates. Current Organic Chemistry, $11,773$. http://dx.doi.org/10.2174/138527207780831747

[8] Shawali, A.S. and Hassaneen, H.M. (1977) ArylAlkanehydrazonates and Their Thio Analogs. Synthesis of Some 2-

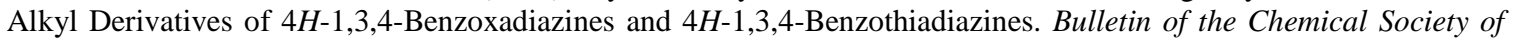
Japan, 50, 2827. http://dx.doi.org/10.1246/bcsj.50.2827

[9] Gouma, S.M., Salah, T.A. and Abdel Hamid, A.O. (2015) Synthesis and Cytotoxicity Evaluation of Some Novel Thiazoles, Thiadiazoles and Pyrido[2,3-d][1,2,4]triazolo[4,3-a]pyrimidin-5(1H)-ones Incorporating Triazole. Monatshefte für Chemie, 146, 149-158.

[10] Gouma, S.M., Ahmed, S.A. and Abdel Hamid, A.O. (2015) Synthesis, Characterization and Pharmacological Evaluation of Some Novel Thiadiazoles and Thiazoles Incorporating Pyrazole Moity As Anticancer Agents. Molecules, 20, 1357-1376.

[11] Hassaneen, H.M., Shawali, A.S., Abdallah, T.A. and Saleh, F.M. (2014) Synthesis and Cycloaddition Reactions of 4,4-dimethyl-2,6-dioxocyclohexane-thiocarboxamides with Nitrilimines. ARKIVOC, 2014, 155-169.

[12] Hassaneen, H.M., Shetta, A.H., Elwan, N.M. and Shawali, A.S. (1982) Reaction of Phenyltrichloromethane with Semicarbazide and Thiosemicarbazide Derivatives. Heterocycles, 19, 1477-1482. http://dx.doi.org/10.3987/R-1982-08-1477

[13] Hassaneen, H.M., Mousa, H.A.H. and Shawali, A.S. (1987) Chemistry of C-Heteroarylnitrilimines. Synthesis and Cycloaddition Reactions of $N$-phenyl-C-(2-thienyl)nitrilimine. Journal of Heterocyclic Chemistry, 24, 1665-1668. http://dx.doi.org/10.1002/jhet.5570240633

[14] Hassaneen, H.M. and Mousa, H.A.H. (1988) C-(2-Thienyl)-N-Arylformohydrazidoyl Chlorides in the Synthesis of Selenadiazolines and Thiadiazolines. Sulfur Letters, 7, 93.

[15] Hassaneen, H.M., Shawali, A.S. and Elwan, N.M. (1990) Synthesis and Cycloaddition Reaction of N-Phenyl-C-Styrylmethanohydrazonyl Bromide. Heterocycles, 31, 247-253. http://dx.doi.org/10.3987/COM-89-5045

[16] Hassaneen, H.M., Shawali, A.S. and Elwan, N.M. (1990) A Convenient Synthesis of 3,5'-Bipyrazolyl Derivatives via Hydrazonyl Halides. Heterocycles, 31, 1041-1047. http://dx.doi.org/10.3987/COM-90-5310

[17] Hassaneen, H.M., Hilal, R.H., Elwan, N.M., Harhash, A. and Shawali, A.S. (1984) The Regioselectivity in the Formation of Pyrazolines and Pyrazoles from Nitrile Imines. Journal of Heterocyclic Chemistry, 21, 1013-1016. http://dx.doi.org/10.1002/jhet.5570210417

[18] Baraldi, P.G., Elkashef, H., Farghaly, A., Vanelle, P. and Fruttarolo, F. (2004) Synthesis of New Pyrazolo[4,3-e]1,2,4-triazolo[1,5-c]pyrimidines and Related Heterocycles. Tetrahedron, 60, 5093-5104. http://dx.doi.org/10.1016/j.tet.2004.04.010

[19] Nagamatsu, T. and Fujita, T. (1999) Facile and General Syntheses of 3- and/or 5-Substituted 7H-pyrazolo[4,3-e]-1,2,4triazolo[4,3-c]pyrimidines as a New Class of Potential Xanthine Oxidase Inhibitors. Chemical Communications, 16, 1461.

[20] Seifi, N., Niaki, M.H.Z., Barzegari, M.R., Davoodnia, A., Zhiani, R. and Kaju, A.A. (2006) Synthesis of 8-aryl-1Hpyrazolo[4,3-e][1,2,4]triazolo[4,3-a] Pyrimidine-4(5H)-imine by Using the Preyssler's Anion [NaP5W30O110 ${ }^{14-}$ as a Green and Eco-Friendly Catalyst. Journal of Molecular Catalysis A: Chemical, 260, 77-81. http://dx.doi.org/10.1016/j.molcata.2006.06.043

[21] Elwan, N.M. (1989) Synthesis and Reactivity of Some Nitrogen Heterocyclic System via Dipolar Cycloadditions. Ph.D. Thesis, Cairo University, Giza City.

[22] Rashad, A.E., Heikel, O.A., El-Nezhawy, A.O.H. and Abdel-Megeid, F.M.E. (2004) Synthesis and Isomerization of 
Thienotriazolopyrimidine and Thienotetrazolopyrimidine Derivatives with Potential Anti-Inflammatory Activity. Heteroatom Chemistry, 16, 226-234. http://dx.doi.org/10.1002/hc.20114

[23] Abdelfattah, B., Kandeel, M.M., Abdel-Hakeem, M. and Fahmy, Z.M. (2006) Synthesis of Certain Fused Thienopyrimidines of Biological Interest. Journal of the Chinese Chemical Society, 53, 403-412.

http://dx.doi.org/10.1002/jccs.200600051 